\title{
A note on risk aversion and herd behavior in financial markets
}

\author{
Jean-Paul Decamps · Stefano Lovo
}

Received: 4 December 2003/ Revised: 4 December 2003

(C) Springer Science + Business Media, LLC 2006

\begin{abstract}
We show that differences in market participants risk aversion can generate herd behavior in stock markets where assets are traded sequentially. This in turn prevents learning of market's fundamentals. These results are obtained without introducing multidimensional uncertainty or transaction cost.
\end{abstract}

Keywords Herd behavior · Risk aversion

JEL Classification $\mathrm{G} 1 \cdot \mathrm{G} 14 \cdot \mathrm{C} 11 \cdot \mathrm{D} 82$

\section{Introduction}

The literature on rational herding pioneering by Bikhchandani, Hirshleifer and Welch [1992] and Banerjee [1992] among others, proves that sequential interaction of rational investors can generate imitative behavior (herding) that prevents learning of the economy's fundamentals. However, in the herding models transaction prices are exogenous and constant, therefore their predictions cannot be directly extended to stock markets. To what extent the endogeneity of trading prices in financial markets can prevent herding phenomena and guarantee full information aggregation?

Avery and Zemsky [1998] (AZ henceforth) and Lee [1998] study the occurrence of herding in stock markets when trading is sequential and prices are endogenous. AZ show that in order to generate herding behavior in a Glosten and Milgrom [1985] style model, it is necessary to introduce multidimensional uncertainty such as, for

J.-P. Decamps $(\triangle)$

GREMAQ-IDEI Université de Toulouse 1, 21 Allee de Brienne, 31000 Toulouse, France, and

Europlace Institute of Finance

e-mail: decamps@cict.fr

S. Lovo

HEC, Finance and Economics Department, 1 Rue de la Liberation, 78351, Jouy en Josas, France e-mail: lovo@hec.fr 
example, event uncertainty or uncertainty on the proportion of informed agents in the economy. Nevertheless, as in Glosten and Milgrom [1985], in the long run all these phenomena vanish and all private information is eventually incorporated into prices. In Lee [1998] an exogenous transaction cost may prevent traders from revealing their private information leading to information aggregation failure.

Within a simple sequential trade model, this note shows that herding, contrarian behavior $^{1}$ and information aggregation failure can occur even in the absence of both multidimensional uncertainty and transaction cost. Differently from AZ, in our model traders and market makers interpret past histories in the same way. Nevertheless, when market makers and traders differ in their risk aversion, the same information affects market makers' quotes and traders' valuations differently. This is sufficient to generate herding, contrarian behaviors and long run informational inefficiency. Section 2 presents the model and our results. Section 3 concludes. Proofs are in appendix.

\section{The model and our results}

We consider a sequential trade model similar to Glosten and Milgrom [1985]: a risky asset is exchanged for money among market makers and traders. At each trading period, a randomly selected trader has a unique opportunity to buy or sell one unit of the asset at the most attractive ask $\left(A_{t}\right)$ or bid price $\left(B_{t}\right)$ respectively. Prices are competitively posted by market makers. We denote with $\mathbf{v}=\mathbf{V}+\varepsilon$ the liquidation value of the asset, where $\varepsilon$ has a normal distribution $N(0, \sigma)$ with $\sigma>0, \mathbf{V} \in\{\underline{V}, \bar{V}\}$ with $\underline{V}<\bar{V}$ and $\operatorname{Pr}(V=\bar{V})=\pi_{0} . \mathbf{V}$ and $\varepsilon$ are independently distributed. Each trader receives a private signal $\mathbf{s} \in\{l, h\}$ with $\operatorname{Pr}(\mathbf{s}=l \mid \mathbf{V}=\underline{V})=\operatorname{Pr}(\mathbf{s}=h \mid \mathbf{V}=\bar{V})=p \in$ $\left(\frac{1}{2}, 1\right)$. Signals are conditionally i.i.d. across traders and independent from $\varepsilon$. Note that we have $\underline{V}<E[\mathbf{v} \mid s=l]<E[\mathbf{v}]<E[\mathbf{v} \mid s=h]<\bar{V}$.

Let $H_{t}$ be the history of trade (past quantities and prices) up to date $t-1$. All agents observe $H_{t}$ and update their beliefs according to Bayes' rule. We denote $\pi_{t}=\operatorname{Pr}[\mathbf{V}=$ $\left.\bar{V} \mid H_{t}\right]$ the public belief at time $t$ and $\pi_{t}^{s}=\operatorname{Pr}\left[\mathbf{V}=\bar{V} \mid H_{t}, s\right], s \in\{h, l\}$, an informed traders' belief at time $t$. A trader's action $\mathcal{A} \in\{$ buy, sell, notrade $\}$ is said to be not informative at date $t$ if it does not affect the public belief that is $\operatorname{Pr}\left[\mathbf{V}=\bar{V} \mid H_{t}, \mathcal{A}\right]=\pi_{t}$. Note that the learning process in the economy regards only the realization of $\mathbf{V}$ and $\operatorname{not} \varepsilon$, still $E\left[\mathbf{v} \mid H_{t}\right]=E\left[\mathbf{V} \mid H_{t}\right]$.

A risk averse agent of our economy has utility function $u(\mathbf{v} x+m)=-\gamma e^{-\gamma(\mathbf{V} x+m)}$, where $x$ and $m$ are respectively the amount of risky asset (inventory henceforth) and money in his portfolio. Thus, in our setting, risk averse agents can differ in their inventory $x$, their cash $m$ and their risk aversion $\gamma$. We assume that the set $\Phi$ of all possible inventories held by the agents in the economy is finite. The distribution of the inventories is exogenous, orthogonal to $\mathbf{v}$ and constant across time. We denote with $f(X)$ the probability that a trader has inventory $x \in X \subset \Phi$. Last, we assume that the set of possible coefficients of risk aversion admits a strictly positive lower bound $\underline{\gamma}>0$.

\footnotetext{
${ }^{1}$ See next section for a precise definition of these behaviors. 
We denote with $\beta$ (resp. $\alpha$ ) the agent's buy (resp. sell) reservation price that corresponds to the asset's price such that this agent is indifferent between buying (resp. selling) one asset or not trading at all. As shown in appendix, the reservation prices for a risk averse agent whose initial inventory is $x$ and that attaches probability $\pi$ to the event $\{\mathbf{V}=\bar{V}\}$ are

$$
\begin{aligned}
& \beta(\pi, x)=\frac{1}{\gamma}\left(-\frac{\gamma^{2} \sigma^{2}(2 x+1)}{2}+\ln \left(\frac{\pi e^{-\gamma \bar{V} x}+(1-\pi) e^{-\gamma \underline{V} x}}{\pi e^{-\gamma \bar{V}(x+1)}+(1-\pi) e^{-\gamma \underline{V}(x+1)}}\right)\right), \\
& \alpha(\pi, x)=\frac{1}{\gamma}\left(-\frac{\gamma^{2} \sigma^{2}(2 x-1)}{2}+\ln \left(\frac{\pi e^{-\gamma \bar{V}(x-1)}+(1-\pi) e^{-\gamma \underline{V}(x-1)}}{\pi e^{-\gamma \bar{V} x}+(1-\pi) e^{-\gamma \underline{V} x}}\right)\right) .
\end{aligned}
$$

We adopt exactly the same definition of herding, contrarian behavior and informational cascade as in Avery and Zemsky [1998]:

A trader with private signal $s$ engages in buy (sell) herding behavior if: (i) initially he strictly prefers not to buy (resp. not to sell); (ii) after observing a positive history of trades $H_{t}$, i.e. $\pi_{t}>\pi_{0}$ (resp. negative history, i.e. $\pi_{t}<\pi_{0}$ ), he strictly prefers to buy (resp. sell).

A trader engages in buy (sell) contrarian behavior if: (i) initially he strictly prefers not to buy (resp. not to sell); (ii) after observing a negative (resp. positive) history of trades $H_{t}$, he strictly prefers to buy (resp. sell).

An informational cascade occurs when the actions of all informed traders are not informative.

Note that if an informational cascade starts at a given date $t$ and never ends then, for any subsequent date, the public belief remains stopped at the level $\pi_{t}$ and trading prices cannot converge to the fundamental value of the asset. Then the market is informational inefficient.

The two following propositions show that the difference in risk aversion between traders and market makers can originate herd, contrarian behavior and informational cascade. As a general rule, herd or contrarian behavior and informational cascade occur when market makers' quotes and traders valuations for the asset react differently to an history of trade. ${ }^{2}$ In our model agents react differently to an history of trade because of the difference in risk aversion between dealers and traders. Take for example, a positive history that increases the public belief $\pi_{t}$. As the public belief $\pi_{t}$ approaches 1 a risk neutral agent's valuation for the asset converges to $\bar{V}$. By contrast, a risk averse agent's buy and sell reservation prices will converge toward levels that are in general different from $\bar{V}$. We now turn to the formal statement of our results.

\subsection{Risk neutral market makers}

We start the analysis with risk neutral market makers and risk averse traders. Bertrand competition among equally uninformed risk neutral market makers leads to bid and ask quotes that are equal to the expected value of the asset given the available information.

\footnotetext{
${ }^{2}$ Based on this remark, in a model where there is exogenous difference in agent's valuation for assets, Cipriani and Guarino [2003] investigate contagion in financial markets.
} 
Thus, time $t$ bid quote, $B_{t}$, is equal to the maximum ${ }^{3}$ of the solutions of the equation

$$
B_{t}=E\left[\mathbf{v} \mid H_{t}, \text { trader sells at } B_{t}\right],
$$

and the ask quote $A_{t}$ is equal to the minimum ${ }^{4}$ of the solutions of the equation

$$
A_{t}=E\left[\mathbf{v} \mid H_{t} \text {, trader buys at } A_{t}\right] .
$$

Direct computations show that solutions of these equations always exist and satisfy

$$
E\left[V \mid H_{t}, l\right]<B_{t} \leq E\left[v \mid H_{t}\right] \leq A_{t}<E[V \mid H, h]
$$

Thus, if time-t-trader has signal $s$ and inventory $x$, then he will buy if $\beta\left(\pi_{t}^{s}, x\right) \geq A_{t}$, he will sell if $\alpha\left(\pi_{t}^{s}, x\right) \leq B_{t}$ and he will not trade elsewhere. We now state our first proposition.

Proposition 1. If traders are risk averse and market makers are risk neutral, then as soon as $\pi_{t}$ is sufficiently close to 1 or to 0 ,

(i) a trader whose inventory is bounded away from $-1 / 2$ and $1 / 2$ will engage in herd or contrarian behavior depending on his initial attitude to buy or to sell the asset;

(ii) if there exist neighborhoods $\mathcal{N}_{-1 / 2}$ and of $\mathcal{N}_{-1 / 2}$ of $1 / 2$ and $-1 / 2$ respectively such that $f\left(\mathcal{N}_{1 / 2}\right)=f\left(\mathcal{N}_{-1 / 2}\right)=0$, then an informational cascade occurs.

Part (i) of Proposition 1 describes individual behavior of risk averse traders. In a setting where market makers are risk neutral and traders are risk averse, we show that, as soon as the public belief $\pi_{t}$ is close to 1 (or to 0 ), a trader whose inventory $x$ is different from $-\frac{1}{2}$ or $\frac{1}{2}$ will choose an action that does not depend on his private signal. Therefore, depending on his initial attitude to buy or to sell the asset, such a trader will engage in herd or contrarian behavior. For instance, a trader who initially would have sold the asset, and whose inventory $x$ satisfies $x<-\frac{1}{2}$, will engage in buy herding behavior after a sufficiently long positive history. The following table summarizes the situations that lead to herding or contrarian behavior as defined in the previous section.

\begin{tabular}{llll}
\hline $\begin{array}{l}\text { Traders' } \\
\text { inventory }\end{array}$ & $\begin{array}{l}\text { Trader's initial } \\
\text { attitude }\end{array}$ & $\begin{array}{l}\text { Positive history } \\
\left(\pi_{t} \text { close to } 1\right)\end{array}$ & $\begin{array}{l}\text { Negative history } \\
\left(\pi_{t} \text { close to } 0\right)\end{array}$ \\
\hline$x<-\frac{1}{2}$ & seller & buy herding & buy contrarian behavior \\
$x>\frac{1}{2}$ & buyer & sell contrarian behavior & sell herding \\
\hline
\end{tabular}

Part (ii) of Proposition 1 explains the relation between long run behavior of prices and the distribution trader's characteristics. The condition on $f$ in part (ii) means that the inventories of all traders in the economy are bounded away from $1 / 2$ and $-1 / 2$.

\footnotetext{
${ }^{3}$ Any other solution would not be an equilibrium as there would exist a larger bid that would provides positive profit to the market makers.

${ }^{4}$ Any other solution would not be an equilibrium as there would exist a smaller ask that would provides positive profit to the market makers.
} 
Thus, from part (i), for a public belief $\pi_{t}$ close to 1 or to 0 , there exist no trader that would submit an informative order. In other words, traders exchange only in order to balance their inventory. These trades do not convey any information as the inventories' distribution is orthogonal to the liquidation value of the asset v. Only in this instance, an informational cascade occurs, bid-ask spread is equal to zero, the public belief does not vary anymore, and prices are steady. Given that the distribution $f$ is constant across time, an informational cascade never ends. Simple computation shows that the thresholds that $\pi_{t}$ must reach in order to trigger a cascade depend on traders's risk aversion and inventories. The smaller the lower bound for risk aversion coefficient $\gamma$ and the closer traders' inventories are to $1 / 2$ or to $-1 / 2$, the more extreme public belief ( i.e., $\pi_{t}$ closer to 1 or to 0 ) are necessary for a cascade to occur. The conditions on the distribution $f$ given in (ii) and $\gamma>0$ imply that all traders in the economy have inventories bounded away from $\overline{1 / 2}$ and $-1 / 2$ and are strictly risk averse. Consequently, a cascade will always happens for $\pi_{t}$ sufficiently close to 0 or to 1 .

\subsection{Risk averse market makers}

We now suppose that two risk averse dealers make a market for risk neutral traders. Consider market maker $i \in\{1,2\}$ at time $t$, and let $B_{t}^{i}, A_{t}^{i}$ and $x_{t}^{i}$ be his bid and ask reservation prices and his inventory respectively. We make the simplifying assumption that market makers are myopic, i.e., when they fix their quotes for the current trade, they do not take into account that they might be lead to trade also in the following periods. Still we assume that in each period market makers set their quotes taking into account the informational content of buy or sell orders that come indeed from informed traders. Consider a risk averse market maker at time $t$ and let $x_{t}^{i}$ be his inventory, then the maximum price that this market maker is willing to pay for one additional unit of the asset is his bid reservation price given that the asset is sold by an informed trader, i.e. $B_{t}^{i}=\beta\left(\operatorname{Pr}\left(\mathbf{V}=\bar{V} \mid H_{t}\right.\right.$, sell $\left.), x_{t}^{i}\right)$. Similarly, the minimum price that this market maker is willing to accept for selling one unit of the asset is his ask reservation price given that the asset is bought by an informed trader, i.e. $A_{t}^{i}=\alpha\left(\operatorname{Pr}\left(\mathbf{V}=\bar{V} \mid H_{t}\right.\right.$, buy $\left.), x_{t}^{i}\right)$. Following Ho and Stoll [1983], as there is no asymmetry of information between the two market makers, in period $t$ both of them will post quotes equal to $A_{t}=\max \left\{A_{t}^{1}, A_{t}^{2}\right\}$ and $B_{t}=\min \left\{B_{t}^{1}, B_{t}^{2}\right\}$. If time- $t$-trader has signal $s$, then he will buy if $E\left[\mathbf{V} \mid H_{t}, s\right] \geq A_{t}$, sell if $E\left[\mathbf{V} \mid H_{t}, s\right]<B_{t}$ and he will not trade elsewhere. We show the following.

Proposition 2. If traders are risk neutral, market makers are risk averse, then as soon as $\pi_{t}$ is sufficiently close to 1 or to 0 and market makers' inventories are bounded away from $\frac{1}{2}$ and $-\frac{1}{2}$,

(i) all traders take the same action and they engage in herding or contrarian behavior depending on their initial attitude to buy or to sell;

(ii) an informational cascade occurs.

As in Proposition 1, proposition 2 describes both individual behavior of agents (part (i)), and long term behavior of prices (part (ii)). We show that if traders are 
risk neutral and market maker risk averse, then expressions (1) and (2) imply that when the public belief $\pi_{t}$ is close to 1 or to 0 , all traders will take the same action no matter the signal they received. For example, when $\pi_{t}$ is close to 1 , all traders will buy (resp. sell) the asset if $A_{t}<\bar{V}$, i.e., $\min \left\{x_{t}^{1}, x_{t}^{2}\right\}>1 / 2$ (resp. $B_{t}>\bar{V}$, i.e., $\max \left\{x_{t}^{1}, x_{t}^{2}\right\}<-1 / 2$ ). Thus, a trader who initially would have sold the asset will engage in buy herding for a public belief $\pi_{t}$ sufficiently close to 1 . The following table provides the different cases of herding and contrarian behavior when market makers are risk averse:

\begin{tabular}{llll}
\hline $\begin{array}{l}\text { Market makers' } \\
\text { inventories at } t\end{array}$ & $\begin{array}{l}\text { Trader's initial } \\
\text { attitude }\end{array}$ & $\begin{array}{l}\text { Positive history } \\
\left(\pi_{t} \text { close to } 1\right)\end{array}$ & $\begin{array}{l}\text { Negative history } \\
\left(\pi_{t} \text { close to } 0\right)\end{array}$ \\
\hline $\min \left\{x_{t}^{1}, x_{t}^{2}\right\}>\frac{1}{2}$ & seller & buy herding & buy contrarian behavior \\
$\max \left\{x_{t}^{1}, x_{t}^{2}\right\}<-\frac{1}{2}$ & buyer & sell contrarian behavior & sell herding \\
\hline
\end{tabular}

With regard to the long term behavior of prices, as in Proposition 1, when the inventory of all risk averse agents in the economy is bounded away form $-1 / 2$ or $1 / 2$, then as soon as the public belief $\pi_{t}$ is sufficiently close to 0 or to 1 , all traders' orders will not be informative and an informational cascade occurs. The difference with proposition 1 is that, when dealers are risk averse, in the presence of an informational cascade, quotes move for inventory purposes even if trades do not conceal any new information. These prices movement might interrupt an informational cascade. Nevertheless, there is no economic force that leads market maker inventories to be exactly equal either to $-1 / 2$ or to $1 / 2$, and so in the long run an informational cascade will occur provided that $\pi_{t}$ is sufficiently close to 0 or to 1 .

\section{Concluding remarks}

Avery and Zemsky [1998] draw the general conclusion of their paper as follows:

"The existence of history-dependent behavior (in either its herd or contrarian form) requires (i) that there exists multiple dimensions of uncertainty, and (ii) that traders' asymmetric information about value uncertainty be sufficiently poor relative to their information about one of the other dimensions of uncertainty."

We show in this note that if traders or market makers are risk averse, neither requirements (i) or (ii) are necessary to obtain herd or contrarian behavior. For what regards the relation between market efficiency and herding, Avery and Zemsky [1998] show that after herd behavior started the flow of information to market makers is not stopped. Thus, in their setting informational cascade never occurs and prices eventually converge to the asset's fundamentals. In our setting, an informational cascade can arises provided that market makers and traders differ in risk aversion and the inventories of risk averse agent are bounded away from $1 / 2$ and $-1 / 2$. If market makers are risk neutral and an informational cascade occurs, buy or sell order do not convey any new information, spread is zero, the public information and prices are constant and therefore market is not efficient. In this instance, traders with different inventory unbalance will take different actions, but no trader will use his private signal to determine the sign of 
his transaction. By contrast, if market makers are risk averse, then during a cascade all risk neutral traders will take exactly the same action, as their inventory plays no role. Moreover, quotes move even in presence of informational cascade because trades change market makers' portfolio composition. This can break herd behavior, and temporally interrupt the informational cascade.

In a related paper (Décamps and Lovo [2004]) we show that information aggregation failure does not relay on the restriction to CARA utility functions nor on the assumption that agents can just choose the sign of their trade but not its size.

\section{Appendix}

\section{Reservation prices}

Take a risk averse agent that attaches probability $\pi_{t}$ to the event $\{\mathbf{V}=\bar{V}\}$ and that holds an amount $m$ of money and $x$ unit of the risky asset. The buy reservation price $\beta\left(\pi_{t}, x\right)$ is the minimum amount of money that this agent is willing to pay in exchange for one additional risky asset. In other words $\beta\left(\pi_{t}, x\right)$ in expression (1) is the $\beta$ that solves

$$
E\left[U(\mathbf{v}, x, m)-U(\mathbf{v}, x+1, m-\beta) \mid H_{t}\right]=0
$$

where

$$
\begin{aligned}
E\left[U(\mathbf{v}, x, m) \mid H_{t}\right] & =E\left[-\gamma e^{-\gamma(\mathbf{V} x+m)} \mid H_{t}\right] \\
& =\pi_{t} E\left[\left(-\gamma e^{-\gamma((\bar{V}+\varepsilon) x+m)}\right)\right]+\left(1-\pi_{t}\right) E\left[\left(-\gamma e^{-\gamma((\underline{V}+\varepsilon) x+m)}\right)\right] .
\end{aligned}
$$

Similarly, the sell reservation price $\alpha\left(\pi_{t}, x\right)$ in expression (2) is the minimum price at which our agent is willing to sell one unit of the asset. That is to say the $\alpha$ that solves

$$
E\left[U(\mathbf{v}, x, m)-U(\mathbf{v}, x-1, m+\alpha) \mid H_{t}\right]=0 .
$$

Taking into account that $\varepsilon$ is normally distributed and that agents have CARA utility function, we have expressions (1) and (2) for the reservation prices.

\section{Proofs of Propositions 1 and 2}

Propositions 1 and 2 are direct consequences of the following lemma

Lemma 1. Take $\pi_{t}$ sufficiently close to 1 or to 0 , and let $x>1 / 2$ and $x^{\prime}<-1 / 2$. Then for any triple of signals $s, s^{\prime}$ and $s^{\prime \prime}$ it results

$$
\begin{gathered}
\alpha\left(\pi_{t}^{s}, x\right)<E\left[V \mid H_{t}, s^{\prime}\right]<\alpha\left(\pi_{t}^{s^{\prime \prime}}, x^{\prime}\right), \\
\beta\left(\pi_{t}^{s}, x\right)<E\left[V \mid H_{t}, s^{\prime}\right]<\beta\left(\pi_{t}^{s^{\prime \prime}}, x^{\prime}\right) .
\end{gathered}
$$


Proof: First, as $\pi_{t}^{l}=\frac{\pi_{t}(1-p)}{\pi_{t}(1-p)+\left(1-\pi_{t}\right) p}$ and $\pi_{t}^{h}=\frac{\pi_{t} p}{\pi_{t} p+\left(1-\pi_{t}\right)(1-p)}, \pi_{t}^{s}$ is continuous in $\pi_{t}$, for $s=h, l$. Second, from expressions (1) and (2), $\alpha$ and $\beta$ are continuous in $\pi$. Third, $\alpha\left(1, \frac{1}{2}\right)=\beta\left(1,-\frac{1}{2}\right)=\bar{V}, \alpha\left(0, \frac{1}{2}\right)=\beta\left(0,-\frac{1}{2}\right)=\underline{V}$ and $\alpha$ and $\beta$ are decreasing in $x$. Then, the results follows from an easy continuity argument.

Proofs of Propositions 1 and 2: Without loss of generality we reason with sell orders. Take an informed trader who strictly prefer not to sell at date 0 . Assume first that the trader is risk averse with inventory $x^{\prime}<-\frac{1}{2}$ whereas dealers are risk neutral. From Lemma 1 , as $\pi_{t}$ is sufficiently close to 0 , we have $\alpha\left(\pi_{t}^{s}, x^{\prime}\right)>B_{t}$ for $s \in\{l, h\}$. Thus, our trader will engage in sell herding and his trade will not be informative. Second, suppose the trader is risk neutral and dealers are risk averse. If at date $t$, the public belief $\pi_{t}$ is close to 0 and dealers inventories satisfy $\max \left\{x_{t}^{1}, x_{t}^{2}\right\}<-\frac{1}{2}$ then, from Lemma $1, B_{t}=\min \left\{B_{t}^{1}, B_{t}^{2}\right\}>E\left[V \mid H_{t}, s\right]$ for $s \in\{l, h\}$ and the trader will engage in sell herding. Once again trades are not informative. Similarly, $\pi_{t}$ close to 1 leads to sell contrarian behavior. Using an analogous argument it can be easily checked that if all risk averse agents' inventories are bounded away from $\frac{1}{2}$ and $-\frac{1}{2}$ then, as soon as $\pi_{t}$ is close to 1 or to 0 , trades are not informative and therefore an informational cascade occurs.

Acknowledgments We would like to thank Bruno Biais, Cristophe Chamley, Thierry Foucault, Christian Gollier and Jacques Olivier for insightful conversation and valuable advice. We would also like to thank the seminar participants at HEC, CentER and Cergy Pontoise University for useful comments. We are grateful to an anonymous referee for helpful suggestions. Financial support from Europlace Institute of Finance is gratefully acknowledged. Of course all errors and omissions are ours.

\section{References}

AVERY, C. and ZEMSKY, P. [1998]: "Multidimensional Uncertainty and Herd Behavior in Financial Markets," The American Economic Review, 88, 724-748.

BANERJEE, A. [1992]: “A Simple Model of Herd Behavior,” Quarterly Journal of Economics, 107, 787818.

BIKHCHANDANI, S., HIRSHLEIFER, D., and WHELCH, I. [1992]: “A Theory of Fads, Fashion, Custom and Cultural Change as Informational Cascade," Journal of Political Economy, 100, 992-1027.

CIPRIANI, M. and GUARINO, A. [2003]: "Herd Behavior and Contagion in Financial Markets," Working paper, Department of Economics, New York University.

DÉCAMPS, J.P. and LOVO, S. [2003]: "Market Informational Inefficiency, Risk Aversion and Quantity Grid," HEC CR 770/2003.

GLOSTEN, L. and MILGROM, P. [1985]: "Bid, Ask and Transaction Prices in a Specialist Market with Heterogeneously Informed Traders," Journal of Financial Economics, 14, 71-100.

HO, T. and STOLL, H. [1981]: "Optimal Dealer Pricing Under Transactions and Return Uncertainty," Journal of Financial Economics, 9, 47-73.

LEE, I.H. [1998]: "Market Crashes and Informational Avalanches," Review of Economic Studies, 65, 741759. 